\title{
LA INTELIGIBILIDAD DE LA NATURALEZA Y SU VINCULACIÓN CON EL CONOCIMIENTO DE LOS UNIVERSALES
}

\author{
THE INTELLIGIBILITY OF NATURE \\ AND ITS NEXUS WITH THE KNOWLEDGE OF THE UNIVERSALS
}

\author{
SEBASTIÁN CONTRERAS \\ Universidad de los Andes (Chile)
}

\begin{abstract}
Resumen: Desde la propuesta de Santo Tomás, el presente trabajo intenta mostrar la vinculación existente entre la inteligibilidad de la naturaleza y el conocimiento intelectual de los universales que pensamos, y que en estricto rigor no existen sino como formas de las cosas singulares que se hallan a nuestro alrededor. Para ello, y partiendo de la idea de que la naturaleza admite ser conocida a causa de su inteligibilidad per se, buscaremos abordar la solución que Santo Tomás entrega a la pregunta por el estatuto metafísico de los universales. Asimismo, y por cuanto se trataría de un caso particular de este citado problema medieval, en la medida que vincula la finitud y contingencia de las tendencias básicas del hombre con la universalidad de ciertos imperativos éticos, nos referiremos también a la cuestión de la ley natural, según la exposición que hace Santo Tomás en su Summa Theologiae.
\end{abstract}

Palabras clave: universales; inteligibilidad de la naturaleza; ley natural; Santo Tomás; filosofía medieval.

\begin{abstract}
Using Aquinas' proposal, this article tries to show the nexus between the intelligibility of Nature and the intellectual knowledge of Universals that we think, and that stricto sensu exist only as forms of singular things that are around us. For it, and based on the idea that Nature allows itself to be known because of its intelligibility per se, we try to show the solution that Aquinas delivers to the question for the metaphysical statute of the universals. Also, and because is a particular case of this medieval problem, since that links the finitude and contingency of the basic inclinations of human person with the universality of certain ethical imperatives, we will also treat the subject of Natural law, in accordance with the exposure of Aquinas' Summa Theologiae.
\end{abstract}

Keywords: Universals; intelligibility of Nature; Natural Law; Aquinas; medieval philosophy.

\section{SUMARIO}

1. Introducción.- 2. Santo Tomás y la solución metafísica al problema de los Universales.2.1. Acerca de la inteligibilidad de la naturaleza (y la "firmeza" metafísica de las esencias en el conocimiento de los universales).- 2.2. Sobre el orden del mundo como fundamento de la inteligibilidad de las cosas.- 3 . La inteligibilidad de la naturaleza y el conocimiento de la ley moral natural.- 4. Consideraciones finales. 


\section{INTRODUCCIÓN}

Asociada a los planteamientos de Porfirio, la pregunta por el tipo de existencia que compete a los universales que pensamos, pasó a convertirse en una de las temáticas centrales de la filosofía medieval, a tal punto que, señala Ramón Guerrero, "fue opinión común entre los primeros estudiosos de la filosofía medieval que el problema escolástico por excelencia, aquel que podía caracterizar toda la filosofía medieval, fue el problema de los universales".

Dicho problema, aún cuando se haya extendido por todo el Medioevo, tuvo su mayor intensidad en un período de poco más de cincuenta años, entre fines del siglo XI y la primera mitad del siglo XII. Y "aunque como cuestión 'medieval' fue puesto en relación con problemas teológicos referentes a las Ideas divinas, tuvo más que ver con el problema del ser, su relación a nuestro conocimiento y la posibilidad de emitir un discurso verdadero sobre él"'. Con todo, y pese a lo intensificado de su tratamiento por parte de los pensadores medievales, ya en Platón y Aristóteles encontramos los primeros intentos de solución a este problema ${ }^{2}$.

Ahora bien, no existen verdaderamente los universales en la realidad, sino tan sólo los entes singulares ${ }^{3}$, a partir de los cuales se posibilita la abstracción y el conocimiento. De modo que se trata el universal de un "signo" de la naturaleza de la cosa, una propiedad de los conceptos según la cual éstos resultan predicables de muchos individuos ${ }^{4}$.

Según esto, nos hemos propuesto un examen de la resolución que entrega la propuesta de Santo Tomás al problema en cuestión, ceñidos, principalmente, de la necesidad intrínseca y la inteligibilidad que ostenta el orden natural. Para ello, nos detendremos tanto en el aspecto gnoseológico como en la perspectiva metafísica de la propuesta de Santo Tomás, que se apoya, respectivamente, en la certeza y estabilidad epistémica que aportan las esencias al conocimiento de la realidad creada, y en la ordenación y necesidad que imprime la Primera Causa al mundo natural. De la misma forma, y buscando articular este planteo con la problemática moral, hemos de sugerir una especial aplicación de este problema de los universales a la exposición que Santo Tomás hace de la ley moral en su Summa Theologiae, en cuanto que se trata de una legalidad que procede ex natura, esto es, a partir de la contingencia ordenada que revisten las inclinaciones básicas del hombre. Pasemos, entonces, al cuerpo de nuestra exposición.

\section{SANTO TOMÁS Y LA SOLUCIÓN METAFÍSICA AL PROBLEMA DE LOS UNIVERSALES}

Siendo un caso particular del llamado problema de la inducción, la cuestión de los universales nos enfrenta a la aparente imposibilidad de dar paso de la realidad creado-contingente de la naturaleza al conocimiento formalmente universal, donde más allá de la simple percepción de los objetos sensibles, hemos de llegar al descubrimiento de las "formas" de las cosas mismas, que duermen tras la pura sensibilidad. Y

\footnotetext{
${ }^{1}$ Rafael RAMón Guerrero, Historia de la filosofía medieval, Madrid, 2002, p. 151 (por ambas citas).

${ }^{2}$ Cfr. José Ferrater Mora, Diccionario de filosofía, Barcelona, 2001, p. 3063 (voz "universales").

${ }^{3}$ SANTO TOMÁs, In Peryhermeneias I, lect. 10.

${ }^{4}$ Cfr. SAnTO TOMÁs, In Metaphysicorum VIII, lect. 13.
} 
es que, sostiene Boecio, "en la percepción de los objetos, los órganos de los sentidos han de recibir las impresiones externas, siendo necesario que la actividad del espíritu sea precedida por una sensación física que atraiga la acción de la inteligencia y despierte las formas en ella dormidas. En estas condiciones, para la percepción, el espíritu no es informado por las sensaciones, sino que juzga por su propia luz sobre los datos que éstas le proporcionan".

Dado este problema, presenta la filosofía de Santo Tomás una solución que integra epistémica y ontológicamente las categorías de "contingencia" y "necesidad", aplicadas éstas a la realidad creada. Retoma, de este modo, la teleología aristotélica, destacando la finalidad y necesidad que Dios ha impreso en el mundo, regularidad que hace posible la ciencia y el conocimiento en general.

Así, el universal expresa para Santo Tomás la participación que tienen varios individuos en una determinada naturaleza. "La naturaleza hombre, en efecto, no se realiza totalmente en un único individuo, pues, en tal caso, habría un hombre único. En la mente, en cambio, la natura hominis adquiere unas propiedades peculiares" ". De esta manera, afirma el Aquinatense, "no se trata [...] de que una naturaleza universal se predique de muchos, sino de que una naturaleza universal sea apta, por sí misma, para ser predicada de muchos" 7 .

De lo expuesto se sigue que la naturaleza universal, que se halla individualizada en la realidad, se encuentra, no obstante, universalizada en la mente. Luego, "considerada en sí misma, tal naturaleza, que fuera de la mente se reviste de singularidad y concreción, es un ser real". Sin embargo, declara Millán Puelles, "lo que no es real es su universalidad, puesto que al existir, aquella naturaleza se contrae siempre a la singularidad y concreción"». Luego, la naturaleza universal es real en concreto, aunque lógica en su universalidad ${ }^{9}$.

Entonces, subyace a la propuesta de Santo Tomás una conciliación entre la existencia finita de la naturaleza, entre su contingencia, y la necesidad e inteligibilidad que en ella se observa, que procede, es cierto, de la ordenación que la Suprema Inteligencia ha dado al mundo, pero que no obstante ello es su constitutivo esencial. Por este motivo, hemos preferido dividir la solución que entrega el tomismo al problema de los universales en i) la cuestión de la inteligibilidad del mundo natural, y ii) la necesidad intrínseca que ostenta la naturaleza, y que pende, en última instancia, de la inteligencia ordenadora de Dios.

\subsection{Acerca de la inteligibilidad de la naturaleza (y la "firmeza" metafísica de las esencias en el conocimiento de los universales)}

Sostiene Santo Tomás que algo es universal en la medida que posee la aptitud para ser predicado de muchos, aunque no sólo respecto del nombre, sino que, fundamentalmente, en razón de lo significado por él ${ }^{10}$, o sea, en cuanto refiere a un ente finito, creado, que dotado de un específico constitutivo formal (su composición esenciaser) es susceptible de ser conocido de modo universal por el entendimiento. De ahí que

\footnotetext{
${ }^{5}$ Cfr. Boecio, De consolatione $\mathrm{V}$, prosa V.

${ }^{6}$ Josep-Ignasi Saranyana, Universal en Ángel Luis GonZÁLEZ. Diccionario de filosofía, Pamplona, 2010, p. 1124.

${ }^{7}$ SANTO TOMÁs, In Peryhermeneias I, lect. 10.

${ }^{8}$ Antonio Millán Puelles, Fundamentos de filosofía. Madrid, 1972, p. 103 (por ambas citas).

${ }^{9}$ J. I. SARANYANA, Universal, p. 1124.

${ }^{10}$ SANTO TOMÁs, In Peryhermeneias I, lect. 10.
} 
el clásico problema de los universales pueda ser presentado, en palabras de José María de Alejandro, como el "de la búsqueda de lo que es a la vez universal y existente, ideal y concreto, válido para siempre y, a pesar de ello, distinto de lo netamente formal"11.

Según esto, postula el de Aquino la efectiva realidad de los universales en tanto que existentes realmente en los individuos particulares, aunque como formas de lo singular. Y esto porque, afirma: "el singular en las cosas materiales, nuestro entendimiento no puede conocerlo directamente y en primer lugar. La razón es que el principio de singularidad en las cosas materiales es la materia individual, y nuestro entendimiento [...] entiende abstrayendo de dicha materia" ${ }^{12}$. Pese a ello, es consciente Tomás de que no puede negarse al intelecto un cierto conocimiento de la realidad concreta, puesto que "no podríamos conocer la comparación del universal con el particular, si no hubiese una potencia que conociera ambos. Así pues, el entendimiento conoce ambas cosas, pero de distinto modo"13.

Por lo tanto, se presenta el problema de los universales como aquél que se pregunta por el tipo de existencia que compete a las quididades universales que pensamos. Y frente a éste, la posición de Santo Tomás es clara: el universal como tal no existe en sí, sino solamente en el espíritu, porque la universalidad de una esencia se deriva de su estado de abstracción. Luego, la esencia que el espíritu concibe de manera intelectual existe efectivamente en la naturaleza creada, y en tal manera, que bien puede afirmarse que el universal halla siempre un fundamento in re. Así, los universales existen, si bien sólo en tanto que formas de las cosas singulares, es decir, en tanto que tienen su fundamento en la realidad concreta, que admite ser conocida.

Todo lo dicho nos sirve de base para afirmar que un primer aspecto considerado por Santo Tomás en su resolución del mencionado problema, no consiste sino en la apelación a la inteligibilidad que ostenta el mundo natural, y en virtud de la cual más allá de la contingencia "existencial" de la naturaleza, se halla en ésta, y gracias a la acción de la Primera Causa, una cierta estabilidad gnoseológica que nos posibilita su conocimiento. De este modo, aún si no existiera entendimiento creado alguno, aún así las cosas serían verdaderas en orden al intelecto divino ${ }^{14}$, que las conoce perfectamente y con entera verdad, o sin posible error, desde siempre y por siempre ${ }^{15}$.

Existe, pues, un principio de inteligibilidad de la naturaleza, presupuesto "lógico y necesario, no sólo pragmático"16, por el cual tanto las cosas naturales como las artificiales son perfectamente aprehensibles por la inteligencia no obstante su individualidad. Ahora, y ante la pregunta por la matriz de la inteligibilidad de las cosas, la respuesta de Santo Tomás es una sola: su esencia.

Por consiguiente, el punto de partida de esta solución no se halla tanto en la esencia abstracta de la metafísica tomista, sino que en la esencia concreta, y específicamente en la esencia concreta singular, aquella que expresa en acto todas las determinaciones del ente particular, tanto las esenciales, como las individuantes, y que es sujeto del acto de ser, precisamente porque por ella y en ella el ente tiene ser ${ }^{17}$. Ahora, tan sólo ésta existe en la realidad, y tan sólo ésta puede ser considerada como matriz de la "certeza" (inteligibilidad) de las cosas. De modo que lo que se quiere significar

\footnotetext{
${ }^{11}$ José María De ALejAndRo, Gnoseología, Madrid, 1974, p. 447.

${ }^{12}$ SANTO TOMÁs, Summa Theologiae I, q. 86, a. 2.

${ }^{13}$ SANTO TOMÁs, In De anima III, lect. 8.

${ }^{14}$ SANTO TOMÁs, De veritate, q. 1, a. 2.

${ }^{15}$ Jesús García López, Metafísica tomista, Pamplona, 2001, p. 362.

${ }^{16}$ Carlos Augusto CASANOva, Reflexiones metafísicas sobre la ciencia natural, Santiago, 2007, p. 90.

${ }^{17}$ SAnto TOMÁs, De ente et essentia I, 5.
} 
con la "certeza de la esencia", es algo así como la firmeza o estabilidad epistémica de una cosa, aquello por lo cual un triángulo es realmente un triángulo, o por lo que la blancura es efectivamente blanca ${ }^{18}$.

Con esto, nos recuerda el tomismo que la esencia "se llama también forma, en cuanto [que] por la forma se significa la certeza de cada cosa, como dice Avicena en [...] su Metafísica"19, porque, como ha sido declarado por Rahner, "la esencialidad concreta de un "existente en sî" [...] es [...] la expresión del área dentro de la cual el esse, en el ente respectivo, como fundamento que es de la realidad de todo, hace ser realmente al ente en cuestión" ${ }^{20}$. En este sentido, la certitudo aviceniana no equivale sino a la "inteligibilidad" metafísica de la que estamos hablando.

Bien, dado que se llama ente a lo que participa de una manera finita en el ser, y esto es lo proporcionado a nuestro intelecto ${ }^{21}$, se sigue que sólo es aprehensible para nuestra inteligencia aquello que posee una quidditas que participe del acto de ser. Por ende, es la essentia rei, en tanto que forma de una materia signada por la cantidad, el fundamento inmediato de la inteligibilidad de una cosa concreta, lo que se explica por lo siguiente: la inteligencia toma por abstracción la forma sin la materia, lo permanente y lo específico, sin lo cambiante y lo individual; y, por eso, aprehende la esencia de un modo abstracto y universal ${ }^{22}$.

Hemos dicho que algo es aprehensible para el intelecto tan sólo en cuanto se halle dotado de una cierta quididad que participe del ser. Y esto que llamamos "ser", no es sino "la actualidad de todos los actos, y por esta razón [...] la perfección de todas las perfecciones" 23 , la causa última de la verdad de las cosas, o mejor, la forma de todas las formas ${ }^{24}$. Este esse, en tanto que lo primeramente conocido por el intelecto, no expresa más que el "estar puesto en la existencia" de las cosas ${ }^{25}$. De modo que, y todavía más profundamente que la esencia, puede decirse de éste ser la causa de la inteligibilidad de las cosas, por cuanto se trata de la primera y más radical de las perfecciones que ha de poseer la cosa concreta-singular, su principio interno de cognoscibilidad, causa de la estabilidad metafísica de todo lo real26. El ser es, de este modo, formalmente inteligible. Dada su condición trascendental, nada escapa a su inteligibilidad: el ser es inteligible en toda su extensión trascendental.

En definitiva, ni la esencia puede ser aprehendida por la inteligencia sino en tanto que refiere a un determinado existente y al acto de ser; ni la existencia puede ser inteligida como tal sino en cuanto imperfeccionada por la esencia, que la ilumina desde el seno de su verdad ontológica, "asidero por donde intencionalmente la asimila la inteligencia humana; la cual, si bien es espiritual y, como tal irreductible a experiencia sensible, sin embargo, no es angélica o puramente espiritual [...] sino encarnada y, por eso mismo, incapaz de alcanzar su objeto formal [...] a no ser por abstracción de las cosas materiales"27, inmediatamente dadas en el conocimiento que procede de la sensibilidad.

\footnotetext{
${ }^{18}$ Jan AERTSEN, La filosofía medieval y los trascendentales, Pamplona, 2003, p. 192.

${ }^{19}$ SANTO TOMÁs, De ente et essentia I, 5.

${ }^{20}$ Karl RAHNER, Espíritu en el mundo. Metafísica del conocimiento finito según Santo Tomás de Aquino, Barcelona, 1963, p. 179.

${ }^{21}$ SANTO TOMÁs, In De causis, lect. 6.

${ }^{22}$ Octavio Nicolás DERISI, Tratado de existencialismo y tomismo, Buenos Aires, 1956, p. 240.

${ }^{23}$ SANTO TOMÁs, De potentia, q. 7, a. 2.

${ }^{24}$ Cfr. SANTO TOMÁs, Summa Theologiae I, q. 3, a. 4.

${ }^{25}$ Cfr. SAnto Tomás, De ente et essentia I, 3.

${ }^{26}$ Alejandro Llano, Gnoseología, Pamplona, 1991, p. 96.

${ }^{27}$ O. N. DERISI, Tratado, p. 250. Entonces, de algún modo u otro, la solución metafísica de Santo Tomás al problema de la inducción parte de una revaloración del conocimiento sensible. Piénsese,
} 


\subsection{Sobre el orden del mundo como fundamento de la inteligibilidad de las cosas}

El conocimiento de los universales se halla fundamentado en la inteligibilidad constitutiva de todas las cosas. De esto hemos hablado ya en la sección anterior. Sin embargo, y todavía más específicamente que la esencia o el acto de ser mismo, la inteligibilidad de las cosas pende de la estructura ordenada y finalizada del mundo natural.

De esta manera, afirma Werner Heisenberg: la naturaleza "está hecha de tal manera [...] que puede ser entendida", porque "las mismas fuerzas ordenadoras que han configurado la naturaleza en todas sus formas son también las responsables de la estructura [...] de nuestra facultad de pensar"28. Luego, no será posible la justificación del conocimiento universal a partir de la realidad creada-contingente si no existe, primero, una cierta necesidad intrínseca, y no puramente derivada, del mundo natural.

Ahora, la naturaleza, entendida como "mundo natural", expresa aquella realidad de una creatura que no obstante su condición de tal, se encuentra dotada de una regularidad y legalidad intrínseca, que, en cuanto a su existencia dependen constitutivamente del influjo del Primer Motor: tanto la naturaleza como el cielo "cuelgan" entitativamente de Dios, sentencia el texto de Metaph XII. Dicho mundo natural, provisto de una estabilidad metafísica y gnoseológica tal que posibilita el conocimiento de los universales, no es sino la manifestación de leyes causales invariables, inexorablemente vinculantes para todos, que convierten a la naturaleza en un reflejo más o menos exacto de la perfección entitativa de Dios. Por este motivo es que la naturaleza siempre se mueve hacia determinados fines, y según un orden específico.

Como puede apreciarse, la naturaleza pasa a transformarse así para Santo Tomás en una vía de conocimiento de Dios ${ }^{29}$. Por esto es que para el Angélico, lo mismo que para la gran mayoría de los pensadores medievales, la naturaleza no será más que un gran libro escrito por la misma Causa Incausada, en donde podemos reconocerla y reconocer gobierno. Prueba de ello es la regularidad que exhibe el mundo natural, por la que Santo Tomás llega a concluir la existencia del Primer Motor. Y es que, declara: "es imposible que cosas contrarias y disonantes convengan siempre o las más de las veces en un orden si alguien no las gobierna, haciéndolas tender a todas y cada una a un fin determinado. Vemos [de este modo] que en el mundo las cosas de naturaleza más diversa convienen en un orden, no casualmente y rara vez, sino siempre o casi siempre. Debe darse, por tanto, alguien por cuya providencia se gobierne el mundo. Y a ese tal llamamos Dios" 30 . Entonces, sin referencia al gobierno divino no puede ser explicado ni el orden ni la inteligibilidad del mundo natural. Por esto es que en opinión de Ana Marta González, la inteligibilidad y finalidad de la naturaleza depende de una realidad no estrictamente natural ${ }^{31}$ : Dios, Causa Incausada de todas las cosas.

Pues bien, se caracteriza esta segunda solución tomista al problema de los universales por su consideración del principio de regularidad del mundo natural (y dicha regularidad es, sin más, el fundamento de su inteligibilidad). Tal es la "necesidad" y "regularidad" que se halla en la naturaleza, que bien ha afirmado Aristóteles que

por ejemplo, en el rol que asigna el Aquinatense al dato que proviene de la sensibilidad (al "testimonio de los sentidos") en sus pruebas racionales de la existencia de Dios.

${ }^{28}$ Werner HeISENBERG, Diálogos sobre física atómica, Madrid, 1975, pp. 126-128 (por ambas citas).

${ }^{29}$ Jorge Martínez BARrera, La política en Aristóteles y Santo Tomás, Pamplona, 2001, p. 110.

${ }^{30}$ SANTO TOMÁs, Contra Gentes I, c. 13.

${ }^{31}$ Cfr. Ana Marta GonZÁlez, Moral, razón y naturaleza, Pamplona, 1998, p. 32. 
aquélla nunca hace nada en vano o sin una cierta finalidad ${ }^{32}$. Por consiguiente, dicha regularidad de la naturaleza, en tanto que expresiva de la ordenación providente de Dios, no exige que cada fenómeno en particular ocurra siempre del mismo modo dondequiera se cumplan ciertas condiciones. Así, y en palabras de Mario Bunge, "la legalidad universal es compatible con excepciones individuales" 33 , motivo por el cual incluso los defectus naturae, cuando se producen, son queridos por Dios en vista de ciertos fines ${ }^{34}$.

Con todo, dicha regularidad de la naturaleza no existe como manifestativa de un mundo increado o eterno, como en la propuesta aristotélica, sino que de un mundo finito-contingente, creado ex nihilo por Dios, el único que puede sacar las cosas de la nada y ponerlas en el ser ${ }^{35}$. De acuerdo con ello, pende la regularidad de la naturaleza de la inteligencia ordenadora de la Primera Causa, porque, como afirmara Boecio, aún el concurso en apariencia accidental de las series de causas depende del orden invariable establecido por la Providencia ${ }^{36}$.

Entonces, dado que todo es obra de una inteligencia ordenadora que ha querido lo necesario como necesario, lo contingente como contingente, y lo libre como $l_{i b r e}{ }^{37}$, se aprecia que la contingencia del mundo, la contingencia de los singulares que posibilitan el conocimiento universal, se trata de una contingencia relativa, no absoluta, en la medida que la realidad creada ha sido impregnada por Dios de una cierta inteligibilidad y necesidad. De esta manera, el contingenismo absoluto de todo lo que no sea la Primera Causa es algo que de ninguna manera es admitido por la metafísica de Santo Tomás ${ }^{38}$ : la realidad contingente, habiendo sido ordenada por la Suprema Inteligencia, se recubre de una cierta necesidad que posibilita su conocimiento ${ }^{39}$.

En suma, gracias a la ordenación que Dios ha impreso en las cosas, existe en la naturaleza una regularidad intrínseca. Según esto, no hay ente tan contingente que en sí mismo no tenga algo de necesario ${ }^{40}$, de manera que si el mundo es contingente, lo es en virtud de una contingencia no total, porque no es puro desorden y caos, sino que cosmos e inteligibilidad constitutiva. Por tanto, es cierto, existe un momento de contingencia en la creación del mundo, porque Dios escoge de entre las esencias potenciales una serie de esencias para su actualización. "Pero como la voluntad divina de crear es inmutable, cada esencia escogida tiene que realizarse con necesidad" 41 .

\section{LA INTELIGIBILIDAD DE LA NATURALEZA Y EL CONOCIMIENTO DE LA LEY MORAL NATURAL}

En las secciones anteriores hemos visto cómo el problema de los universales se resuelve apelando a una naturaleza de suyo ordenada y dotada de inteligibilidad. Dicha naturaleza, expresión de la ordenación que Dios ha impreso en las cosas, se presenta para Santo Tomás como la causa de la "certeza" gnoseológica que se halla en

\footnotetext{
${ }^{32}$ ARISTÓteles, Politica I, 2, 1253a 9.

${ }^{33}$ Mario Bunge, Causalidad, Buenos Aires, 1961, p. 33.

${ }^{34}$ Étienne GILSON, El espíritu de la filosofía medieval, Madrid, 1981, p. 338 (por ambas citas).

${ }^{35}$ Cfr. SAnto Tomás, Summa Theologiae I, q. 45, a. 2; Contra Gentes III, c. 66.

${ }^{36}$ BOECIO, De consolatione $\mathrm{V}$, prosa 1 .

${ }^{37}$ SAn Agustín, De civitate Dei V, 9, 3.

${ }^{38}$ Cfr. Ángel Luis GonZÁLEZ, Teología natural, Pamplona, 1995, p. 133.

${ }^{39}$ Cfr. SANTO TOMÁs, Summa Theologiae I, q. 2, a. 3.

${ }^{40}$ SANTO TOMÁs, Summa Theologiae I, q. 86, a. 3.

${ }^{41}$ Josef ESTERMAnN, Historia de la filosofía, Lima, 1997, p. 93.
} 
lo real, "firmeza" que se patentiza al espíritu a través de las esencias de las cosas (y, según hemos dicho, de las esencias concretas singulares).

Ahora, esta naturaleza funda asimismo el conocimiento moral, en donde también opera una cierta forma de inducción (que es el procedimiento intelectual que permite dar paso del dato sensible-contingente al conocimiento intelectual). Según veremos a continuación, la argumentación tomista en torno al problema de la ley natural viene a presentarse como un caso especial de la llamada cuestión de los universales, en tanto que también en ella se halla en juego el paso desde lo finito-contingente a lo necesario-universal.

Pues bien, la lex naturalis, en cuanto participación de la ley eterna en la criatura racional $^{42}$, no es más que la ley de la naturaleza (esencia) humana, en tanto que efectivamente conocida por el hombre a partir de sus tendencias básicas y naturales. Y todavía más, la ley natural es la propia naturaleza humana "en cuanto ordena al hombre a su fin último, porque es la [...] ley eterna considerada en el hombre como en sujeto pasivo, regulado y medido por ella"43.

Ahora, "por naturaleza humana debemos entender "aquella constitución o estructura fija en el hombre que es a la vez permanente y estable en medio de las mudanzas a las que puede verse sometido". Es la esencia, aquello por lo que el hombre es y se comporta como tal, es algo invariable, permanente e identificador del propio hombre. La naturaleza humana es la índole propia del ser humano, su peculiar esencia o manera de ser, en tanto que efectivamente dada en él como su más radical principio activo" ${ }^{4}$ Dado esto, de la propia naturaleza del hombre surge la regla fundamental de su obrar, porque siendo la naturaleza lo que constituye al hombre como tal (su esencia), es a la vez el criterio de lo que al hombre le corresponde hacer como tal ${ }^{45}$.

Entonces, y ante la pregunta por la vinculación entre ley natural y naturaleza del hombre, la única respuesta posible es la siguiente: la ley natural manifiesta en forma de deberes las exigencias (naturales) que la propia naturaleza del hombre le dicta ${ }^{46}$. La razón de ello, el mismo "es" de la naturaleza humana tiene inscrito un "debería" 47 , porque la naturaleza del hombre lleva inscrita una serie de tendencias que a éste se le presentan como buenas y perfectivas de su ser.

Sin embargo, no es de extrañarnos que la llegada del modernismo haya traído consigo, tanto un vuelco en la consideración del proceso cognoscitivo, como una separación radical de las categorías de ser y deber, y junto con ello, un cambio en la comprensión teórica de la naturaleza, que ya no se ha de entender más que como un mero factum brutum, y no como una realidad normativo-metafísica, fundamento del bien humano.

Es en este contexto donde la apelación a la llamada falacia de Hume se ha tornado especialmente recurrente. Según ésta, es imposible la derivación del bien desde la simple descriptibilidad del ser, de modo que el dictum de la ética humeana no es sino la imposibilidad de fundar el bien moral en la naturaleza, entendida ésta como un puro conjunto de hechos. Así, declara el autor: "en todo sistema moral de que haya tenido noticia, hasta ahora, he podido siempre observar que el autor sigue durante cierto tiempo el modo de hablar ordinario, estableciendo la existencia de Dios o realizando observaciones sobre los quehaceres humanos, $\mathrm{y}$, de pronto, me encuentro

\footnotetext{
${ }^{42}$ SANTO TOMÁs, Summa Theologiae I-II, q. 91, a. 2.

${ }^{43}$ José Joaquín UGARTE, Curso de filosofía del derecho, Santiago de Chile, 2010, p. 418.

${ }^{44}$ Mauricio BeUCHOT, Derechos humanos y naturaleza humana, México, 2000. p. 61.

${ }^{45}$ Javier HERVADA, Introducción crítica al derecho natural, Pamplona, 1998, p. 143.

${ }^{46}$ M. BeuCHOT, Derechos, p. 69.

${ }^{47}$ Henry Veatch, Natural law and the 'Is'- 'Ought' question, 'Catholic Lawyer”, 26 (1981), p. 265.
} 
con la sorpresa de que, en vez de las cópulas habituales de las proposiciones: es y no es, no veo ninguna proposición que no esté conectada con un debe o un no debe. Este cambio es imperceptible, pero resulta, sin embargo, de la mayor importancia. En efecto, en cuanto que este debe o no debe expresa alguna nueva relación o afirmación, es necesario que ésta sea observada y explicada y que al mismo tiempo se dé razón de algo que parece absolutamente inconcebible, a saber: cómo es posible que esta nueva relación se deduzca de otras totalmente diferentes" 48 .

La posición de George Edward Moore es todavía más crítica de la filosofía de Santo Tomás, sobre todo en lo que se refiere a su rechazo de las éticas con "sustento metafísico". Sobre éstas, señala: "cada vez que se mantiene que, de cualquier proposición que afirme "la realidad tiene esta naturaleza", puede inferirse o confirmarse cualquier otra proposición que afirme "esto es bueno en sí mismo", se comete falacia naturalista", y en este sentido, todos aquellos que definen la bondad moral en términos metafísicos declaran, "implícita o explícitamente, que el conocimiento de lo real suministra algunas razones para mantener que ciertas cosas son buenas en sí mismas"49: la verdad de la proposición "esto es un bien en sí mismo" no puede ser, entonces, reducida a ninguna aserción acerca de la realidad.

Pese a lo convincentes que pudieran resultarnos tales refutaciones a la propuesta de la filosofía clásica del derecho y la ley natural, lo cierto que en ellas mismas subyace la matriz de su propia deficiencia: ambas parten de una naturaleza puramente existencial y fenoménica, concebida ésta como el simple objeto de las ciencias naturales. Y claro. De una estructura no-normativa, como lo sería la naturaleza de la filosofía de Moore, mal podría obtenerse una determinación de lo bueno o recto en sentido moral. Por ello es, justamente, que en opinión de Moore la naturaleza no tiene relevancia en la dilucidación del bien humano.

Volviendo a la temática central de nuestra exposición, a saber, el problema de los universales y su vinculación con la inteligibilidad de la naturaleza, representa el conocimiento de la ley natural una variante específica de la llamada cuestión de los universales, precisamente porque nunca habría razones suficientes para justificar las proposiciones universales de índole moral, como el primer principio práctico de la filosofía tomista, en la mera contingencia del mundo y la realidad finito-creada. En contra de ello, declara Santo Tomás que se fundamenta el bien humano en la consideración de una naturaleza normativa, que determina, por medio de las inclinaciones básicas del hombre, los criterios teórico-prácticos de la perfección humana. Intentaremos, de este modo, y a partir de la exposición tomista de la lex naturalis, vislumbrar la solución que entregaría Santo Tomás a la problemática de la inteligibilidad de la naturaleza en el plano de la moralidad.

Lo primero que se ha de precisar es lo siguiente: el fundamento objetivo de la moralidad humana no consiste en el simple atenerse a la naturaleza entendida ésta como pura y neutra facticidad, ya que si tratamos de aplicar este sentido de naturaleza a la esfera moral, veremos inmediatamente que es imposible ${ }^{50}$. En segundo término, el conocimiento de la ley natural se halla anclado a la existencia de ciertas operaciones que de suyo le convienen a la naturaleza humana, aunque no por el mero acuerdo o convención, sino que por su conformidad con el orden natural ${ }^{51}$. En este sentido, no es cierto que entre el plano del ser y el ámbito del bien no exista más vínculo que aquél que procede de una relación accidental (de mera contingencia), que no hace posible el

\footnotetext{
${ }^{48}$ Hume, Treatise III, 1, 1, nn. 469-470.

${ }^{49}$ MoORE, Principia Ethica IV, 67 (por ambas citas).

${ }^{50}$ Dietrich Von HILDEBRAND, Ética, Madrid, 1983, p. 185.

${ }^{51}$ SANTO Tomás, Contra Gentes III, c. 129.
} 
planteamiento de una moral naturalista. Al contrario, precisamente porque la norma, lo normante, "radica en la naturaleza" 52 es que es posible la obtención de juicios universales sobre el bien humano a partir de la consideración del orden natural.

Por tanto, sostiene Santo Tomás que "todas las cosas hacia las que el hombre siente inclinación natural son aprehendidas naturalmente por la inteligencia como buenas y, por consiguiente, como necesariamente practicables" ${ }^{53}$. De este modo, se comportan las tendencias naturales como la ratio cognoscendi de esta ley de la naturaleza, que, en tanto que descubierta por la actividad de la razón, no nos muestra sino las determinaciones objetivas de lo bueno moral (sólo así se entiende el hecho de que el hombre se nos aparezca, en palabras de Antonio Millán Puelles como un ser en el que ciertas necesidades materiales son, a la vez, necesidades morales ${ }^{54}$ ).

Pues bien, exhibe la naturaleza, en tanto que causa ordinationis que se halla en las $\operatorname{cosas}^{55}$, tres niveles de tendencias a las que el hombre se encuentra sujetado por su condición de animal racional, referidas tanto a la conservación de su ser y la perpetuación de la especie, como al conocimiento de la verdad y al ejercicio de la sociabilidad. Así, existe primero una cierta inclinación al bien según la naturaleza que el hombre tiene en común con todas las substancias, y a causa de la cual apetece la conservación de su naturaleza. Junto a ella, "existe en el hombre una inclinación hacia algo más específico, según la naturaleza que tiene en común con los restantes animales. Y [...] en tercer lugar existe en el hombre una inclinación al bien según la naturaleza de la razón, que es la suya propia, de modo que el hombre tiene inclinación natural a conocer la verdad acerca de Dios y a vivir en sociedad" 56.

Por tanto, pertenecen a la ley natural, en primer lugar, ciertos principios comunísimos que son de todos conocidos, y que no admiten excepción alguna. Tales son sus particularidades, que no cabe a su respecto que la ley de la naturaleza pueda ser borrada del corazón humano: dichos principios, que la propia naturaleza enseña ${ }^{57}$, son per se nota omnibus, dice Santo Tomás. Existen, también, una serie de conclusiones próximas y remotas de los principios primarios, que originan, tanto los preceptos de orden secundario, como aquellos de orden terciario ${ }^{58}$, y que además de poder variar en algunas situaciones, no son conocidas por todos los hombres de la misma manera que los principios primarios de derecho natural.

Según esto, el conocimiento de la ley moral procede ex inclinatione hominis in proprium finem, donde cada inclinación sigue a una forma, y donde toda forma está provista de una determinada inclinación ${ }^{59}$. Dicho esto, tal es la legalidad que ostentan las inclinaciones básicas del hombre, que el orden moral se origina, justamente, en el mundo de las cosas o de la realidad objetiva, en el llamado "mundo natural", presentándose, por ello, como un orden del ser legible en la naturaleza. Así, el bien del ser humano no sería otro que aquél que es descubierto por la actividad de la razón en la contemplación de lo real, de suerte que lo justo natural "no es, en verdad, inventado

\footnotetext{
52 Javier HERVADA, Historia de la ciencia del derecho natural, Pamplona, 1996, p. 157.

${ }^{53}$ SAnto Tomás, Summa Theologiae I-II, q. 94, a. 2. Conforme con ello, se presenta el orden moral como paralelo al orden de las inclinaciones inscritas en la naturaleza humana.

${ }^{54}$ Antonio Millán Puelles, Persona humana y justicia social. Madrid, 1982, p. 12.

${ }^{55}$ Cfr. SANTO TOMÁs, In Physicorum VIII, lect. 3.

${ }^{56}$ SANTO TOMÁs, Summa Theologiae I-II, q. 94, a. 2.

${ }^{57}$ Cfr. JUSTINIANO, Digesto I, $1,1$.

${ }^{58}$ Cfr. Santo Tomás, Summa Theologiae I-II, q. 94, a. 6.

${ }^{59}$ SAnTO TOMÁs, Summa Theologiae I-II, q. 8, a. 1.
} 
por la razón, sino simplemente conocido por ella"60, a diferencia de lo propuesto por el neokantismo o la teoría neoclásica de la ley natural.

En definitiva, el conocimiento de la ley natural supone el conocimiento de la naturaleza humana y de las inclinaciones del hombre, así como el conocimiento de la existencia de Dios y de la relación de absoluta dependencia del hombre respecto de É $l^{61}$, básicamente porque el carácter normativo de la ley natural tiene como su fundamento último, lo mismo que la inteligibilidad de las esencias, no a la regularidad inmanente de los procesos naturales, sino que a la realidad de lo real, Dios, Causa Primera del orden del mundo.

Por último, la aplicación del problema de los universales, o del problema de la inducción, a la cuestión de la ley natural es clara: ex ante, pareciera que no es posible fundar el conocimiento moral en la pura contingencia y "desorden" del mundo natural (o de la naturaleza humana). Sin embargo, y en esto hemos sido insistentes, no es la naturaleza de Santo Tomás un puro conjunto de hechos sin más vinculación que el azar. Todo lo contrario. Se trata de un principio que mueve al hombre con carácter normativo y que éste descubre a través de la regularidad de sus tendencias. Entonces, y porque la naturaleza es para Santo Tomás la matriz de la inteligibilidad de las cosas, incluso el conocimiento moral puede ser fundamentado en ella. Y esto es clave en la consideración de las inclinaciones naturales como materia a partir de la cual despunta el conocimiento de los preceptos de la ley moral, porque éstas, a pesar de su contingencia y finitud, sirven de base para la formulación de los imperativos naturales de moralidad, que la inteligencia (nous) induce a partir de los datos de la sensibilidad: la tendencia a la conservación, la tendencia a la procreación, la tendencia al conocimiento de la verdad y al ejercicio de la sociabilidad. Esto no sería posible si la naturaleza humana fuera un caos, y en este caso no quedaría más posibilidad para fundamentar el conocimiento moral que la ley de la razón práctica de Kant, o los bienes humanos básicos de Finnis o Gómez-Lobo.

Bien puede sostenerse, así, una conjugación epistemológica entre la realidad finito-contingente (pero ordenada por la Suprema Inteligencia) de las inclinaciones básicas del hombre, y las verdades morales universales como el primer principio de la razón práctica que ordena hacer el bien y evitar el mal. La razón de ello, existe en la naturaleza un elemento de certeza y estabilidad gnoseológica, que posibilita la fundamentación del conocimiento moral a partir de las tendencias humanas. En esto consiste la tesis tomista sobre el conocimiento de la legalidad que procede de la naturaleza. Baste por ahora lo que acá hemos señalado.

\section{CONSIDERACIONES FINALES}

Según ha podido apreciarse, frente al llamado problema de los universales, ha presentado la síntesis tomista una solución tal que logra conciliar, por una parte, la condición creada y finita del mundo natural, y, por otra, la necesidad constitutiva que éste ostenta. Sólo así ha podido justificar razonablemente el paso desde la contingencia de las cosas particulares a la universalidad del conocimiento intelectual. Luego, y por una suerte de inducción, el intelecto alcanza al conocimiento del universal desde el dato de la sensibilidad. Es en este contexto que la metafísica de Santo Tomás ha postulado la existencia de un orden permanente y uniforme en la naturaleza, que,

\footnotetext{
${ }^{60}$ Alejandro SILVA BASCuÑán, Tratado de derecho constitucional. Principios, Estado y Gobierno, Santiago de Chile, 1997, p. 13.

${ }^{61}$ José Joaquín UGarTe, El derecho de la vida, Santiago de Chile, 2006, p. 53.
} 
no obstante su contingencia (en cuanto creatura), exhibe tal estabilidad metafísica y necesidad que permite la obtención de conocimientos verdaderos y universales que hacen posible la ciencia y la tarea filosófica ${ }^{62}$.

Conforme con ello, bien pueden las determinaciones formales de una quidditas ser representadas universalmente por un mismo concepto, de modo que el universal, "en cuanto a lo que es (id quod), existe real, aunque fundamentalmente, en las cosas singulares"63. De esta manera, y una vez abstraída del ente concreto, la esencia se nos presenta como un contenido inteligible común a muchos, esto es, como un universal. Así, sólo en la medida que seamos capaces de trascender el caso individual hic et nunc, llegaremos a comprender la naturaleza de la cosa ${ }^{64}$. De ahí que los universales expresen la naturaleza de un algo real, no obstante que su carácter de universalidad proceda directamente de la mente (tanto de la mente de Dios, que es causa de todo lo creado, como de la mente finita, que conoce lo creado en tanto que participa en el orden del ser). Se entienden así las palabras de Santo Tomás en orden a comprender que la esencia a la que le ocurre, o ser entendida, o ser abstraída, "no existe salvo en las cosas singulares", sin perjuicio de que "el ser entendido o el ser abstraído o la intención de universalidad existan en el intelecto" ${ }^{65}$.

Ahora bien, y porque el fundamento del conocimiento universal se halla en la inteligibilidad y necesidad de la naturaleza, lo que se está en juego para una adecuada comprensión de la resolución tomista al problema de los universales no es más que la adecuada comprensión del concepto de physis. A lo largo de este trabajo hemos apelado a dos sentidos distintos de naturaleza, según si hemos querido referirnos a la inteligibilidad intrínseca de todo lo creado, y en este caso hemos hablado de "naturaleza" como "mundo natural", o según si consideramos que la naturaleza misma es basamento del conocimiento moral, en cuyo caso hemos tratado más bien de la "naturaleza" como "esencia del hombre en cuanto principio del obrar".

En cuanto al primero de estos sentidos, si el mundo natural está ordenado, y si en éste encontramos una cierta regularidad, ni el orden del mundo, ni la posibilidad de una naturaleza en general que sea normativa, pueden ser explicados con prescindencia de la existencia de Dios. Porque es Dios, en definitiva, el fundamento del orden de la realidad y del mundo natural, que no es pura y neutra facticidad (una materialidad ciega, diría Joseph Ratzinger ${ }^{66}$ ), sino que una realidad metafísico-normativa per se, que constitutivamente se halla cargada de deber, para emplear la expresión de Mauricio Beuchot ${ }^{67}$ (prueba de esto último son las propias palabras de Santo Tomás, en su In Ethicorum: es la propia naturaleza, en tanto que creada por Dios, la que ha impreso en la mente de los hombres una idea de lo bueno y de lo justo).

En cuanto al sentido de naturaleza entendida ésta como esencia, se recogen a propósito de éste dos ideas básicas: "la primera -dice Juan Manuel Burgos- es que las cosas naturales tienen un modo de ser [...] estable y con una estructura dada y fijada: la esencia. La segunda es que este modo de ser no es estático, sino dinámico: los seres naturales poseen un principio activo que les orienta y les empuja hacia su perfección que consiste en desarrollarse según los patrones correspondientes a su modo de ser" ${ }^{68}$. Tan sólo este sentido, la naturaleza como esencia dinámica, permite

\footnotetext{
${ }^{62}$ Cfr. Alistair Cameron CROMBIE, Medieval and early modern science I, New York, 1959, p. 5.

63 J. M. De Alejandro, Gnoseología., p. 460.

${ }^{64}$ Juan José SAnguineti, Lógica. Pamplona, 1985, p. 43.

${ }^{65}$ SANTO TOMÁs, Summa Theologiae I, q. 85, a. 2 (por ambas citas).

${ }^{66}$ Joseph RATZINGER, Verdad y libertad, "Humanitas", 14 (1999), p. 206.

${ }^{67}$ Cfr. Mauricio Beuchot, Filosofía y derechos humanos, México, 1993, p. 146.

${ }^{68}$ Juan Manuel Burgos, Repensar la naturaleza humana, Madrid, 2007, p. 29.
} 
una adecuada consideración del bien humano a partir del orden objetivo de la realidad, que no procede, como lo ha estimado el kantismo, de una aislada actividad de la razón humana, sino que de la ordenación que ha dado al mundo la Suprema Inteligencia ${ }^{69}$, Causa Primera por cuya providencia se gobierna todo lo real.

Con todo, y porque Santo Tomás se estaría refiriendo a uno y otro sentido de la voz "naturaleza" en su fundamentación del conocimiento universal, sea que se trate del conocimiento teórico de las esencias, sea que se trate del conocimiento práctico de la ley moral, hemos de caracterizarlas, a ambas, de la siguiente forma. Primero, en ambos casos estamos frente a realidades metafísicas, no estrictamente empíricas. Segundo, en ambos casos, la naturaleza es también una realidad trascendente, que no se halla clausurada sobre sí, sino que mira, como a su objeto de deseo, al propio Dios. Tercero, ambos sentidos de naturaleza nos enfrentan a una instancia de apelación normativa, de donde bien puede ser deducido el conocimiento moral. Y cuarto, tanto la naturaleza-mundo natural, como la naturaleza-esencia, son presentadas por Santo Tomás como realidades teleológicas, en tanto que ambas se hallan dotadas de una cierta finalidad que les es esencial.

Por último, no es del todo concluyente sostener que el conocimiento de los universales se encuentra fundamentado en la misma realidad de lo creado, en la naturaleza. Y no porque no sea así en la propuesta de Santo Tomás, sino porque esto nos pone de frente a otro problema: el de si esta realidad creada (a la que hemos llamado indistintamente "naturaleza", "orden natural") posee o no una inteligibilidad per se. Esto, que pareciera un asunto de menor importancia, no lo es tal, por ejemplo en la fundamentación de la inmutabilidad de la ley natural, porque si la naturaleza, que es contingente en su ser, no se halla dotada de una necesidad intrínseca, no habría razón alguna para considerar como incambiantes las llamadas verdades morales: lo bueno moral podría dejar de serlo por una determinación arbitraria de Dios.

Entonces, o afirmamos que no obstante su carácter de creatura ostenta la naturaleza una necesidad per se, o sucumbimos ante la posibilidad de que el propio Dios, que es causa de este orden del mundo (como pudo serlo de cualquier otro orden que hubiese dispuesto), pueda hacer que lo bueno se convierta en malo, o que lo malo se haga bueno, o, lo que es lo mismo, que una tal y cual cosa deje de ser tal. De este modo, afirmar que el conocimiento de los universales, y, por cierto, de la ley moral, se halla fundado en la naturaleza misma, nos exige no sólo que la realidad creada esté (extrínsecamente) dotada de necesidad. Nos exige, sobre todo, que dicha realidad sea caracterizada como necesaria en sí. De lo contrario, nada impediría que la voluntad divina pudiera intervenir en la historia para modificar las esencias de las cosas (y, como ya lo hemos dicho, son las esencias de las cosas las que les otorga su "certeza", metafísicamente hablando).

Por tanto, "si aceptamos que absolutamente todo lo creado y en todos sus aspectos depende de la libertad de Dios, debemos reconocer también que las mismas naturalezas de las cosas y las propiedades que les corresponden carecen de necesidad en su raíz, por más que Dios libremente las dote de estabilidad en su ser y en su obrar"70. Por este motivo, es cierto que la necesidad de la naturaleza es, de algún modo, una necesidad refractada, en el sentido de que "todo lo que es, en un sentido

${ }^{69}$ Sebastián CONTRERAS, Metafísica, naturaleza y orden moral, "Persona y Sociedad", 24/1 (2010), p. 70.

${ }^{70}$ Santiago ORREGo, Fundamentación metafísica de la inmutabilidad de la ley natural en La ley natural como fundamento moral y jurídico en Domingo de Soto, (Juan CRUZ CRUZ, ed.), Pamplona, 2007, p. 70. 
cualquiera, debe necesariamente su ser a Dios"71; pero no significa ello que en la naturaleza misma no exista una necesidad per se, en cuyo caso no podría postularse, bajo ningún respecto, la "firmeza" gnoseológica de la que hemos venido hablando. De manera que ha de darse algo que en las cosas sea el dato inmediato de su necesidad: las esencias, que, en palabras de Domingo de Soto, son eternas y no tienen causa ${ }^{72}$ (y "no tienen causa", explica Santiago Orrego ${ }^{73}$, porque "no depende de la acción creadora de Dios el que a una determinada esencia le correspondan determinados atributos esenciales").

Así, y tal y como ha sido testificado por Gilson ${ }^{74}$, Santo Tomás, lo mismo que todos los pensadores medievales, se apoya siempre en la observación de una necesidad para deducir de ella el conocimiento universal. Porque cada vez que es posible comprobar la existencia de una constancia, de algo que sucede ut in pluribus, podemos estar seguros de que esa constancia tiene una causa, y esa causa no puede ser sino la presencia de una naturaleza. Tal es la importancia de este asunto, que el existir mismo de la ciencia sería imposible si la naturaleza no mostrara una cierta regularidad ${ }^{75}$. Luego, en la propia regularidad de la naturaleza se descubre su necesidad misma ${ }^{76}$.

En fin, en esto consiste la salida metafísica de Santo Tomás al problema de los universales, en que éstos, en estricto sentido, existen tan sólo en las cosas singulares, y en que si en éstas no hubiese una matriz de inteligibilidad per se, entonces no sería posible dar paso del concreto singular al universal formado por nuestro entendimiento, $\mathrm{y}$, lo que es peor, tampoco sería posible el conocimiento humano en general, porque, como lo ha mostrado toda la tradición clásica, en el origen mismo del conocimiento intelectual siempre se halla un particular-concreto en el que la inteligencia debe advertir la forma misma de la cosa oculta tras la sensibilidad. Y claro, si en el particular-concreto no existe matriz alguna de inteligibilidad, por mucho que la inteligencia intente acceder a la realidad tras la percepción, no podrá nunca alcanzar el universal desde el simple estar de frente a la realidad sensible.

Fecha de recepción del artículo: Marzo 2010

Fecha de aceptación y versión final: Diciembre 2010

${ }^{71}$ SANTO TOMÁs, Comp Theologiae I, c. 68 (por ambas citas).

${ }^{72}$ Domingo DE Soto, Summulae $35 \mathrm{r}$.

${ }^{73}$ S. ORREGO, Fundamentación metafísica, p. 77.

${ }^{74}$ E. GILSON, El espíritu de la filosofía, p. 334.

${ }^{75}$ Cfr. Mariano ArTigas, La mente del universo, Pamplona, 2000, pp. 60 ss.

${ }^{76}$ Cfr. SAnTo Tomás, In Physicorum VIII, lect. 8. 